\title{
L'ESPRIT FÉMININ DU SIÈCLE DES LUMIÈRES DANS LES \\ LETTRES ESPAGNOLES : Louise d'Épinay, Anne-Thérèse de Lambert et Marie de Rabutin-Chantal (Madame de Sévigné)
}

\author{
THE WOMEN'S STUDIES OF THE AGE OF \\ ENLIGHTENMENT IN SPANISH LETTERS: Louise d'Épinay, \\ Anne-Thérèse de Lambert and Marie de Rabutin-Chantal (Madame de \\ Sévigné)
}

ONANDÍA RUIZ, Beatriz ${ }^{1}$

\begin{abstract}
RÉSUMÉ : Louise d'Épinay, Anne-Thérèse de Lambert ou Marie Rabutin Chantal, plus connue sous le nom de Madame de Sévigné, sont des auteures dont la réception marqua un tournant au sein des cercles les plus élitistes de l'Espagne du XVIII ${ }^{\mathrm{e}}$ siècle, et plus particulièrement pour les lectures qui étaient celles des femmes espagnoles de l'époque. Des lectures qui, depuis des temps immémoriaux, étaient caractérisées par une tendance morale et religieuse notoire, ainsi que par le martèlement des valeurs traditionnelles et patriarcales inculquées dès le plus jeune âge au genre féminin. Pour toutes ces raisons, ce travail s'intéressera à la fortune littéraire et à la réception de ces trois pédagogues françaises en Espagne, ainsi qu'à la traduction de leurs œuvres, à leur influence sur certaines intellectuelles espagnoles de l'époque et aux différentes versions qui en furent publiées tout au long de de la période des Lumières en Espagne.
\end{abstract}

MOTS-CLÉS : France-Espagne, XVIII ${ }^{\mathrm{e}}$ siècle, pédagogie, traduction, femmes.

\begin{abstract}
The Spanish reception of authors such as Louise d'Épinay, Anne-Thérèse de Lambert or Marie Rabutin Chantal, better known as Madame de Sévigné, had a before and after in the most elite circles of the Eighteenth century Spain and especially in the readings of Enlightenment Hispanic women. Readings, which from time immemorial, were characterized by a flagrant religious morality and by the continual repetition of traditional and patriarchal values, imposed on the feminine gender from an early age. For all this, this work will focus on the literary fortune and the Spanish reception of these three French pedagogues, as well as their translations, their influence on some Spanish intellectuals of the time and the different versions published throughout Hispanic Lights.
\end{abstract}

KEYWORDS: France-Spain, 18th century, pedagogy, translation, women.

\footnotetext{
${ }^{1}$ Profesora Adjunta Universidad del País Vasco-Euskal Herriko Unibertsitatea. E-mail: beatriz.onandia@ehu.eus Jangada | nr. 12, jul/dez, 2018 | ISSN 2317-4722

5 | P ág i n a
} 
Le XVIII ${ }^{\mathrm{e}}$ siècle fut une période privilégiée et féconde eu égard aux traductions remarquables qui parurent alors. Cette pratique connut un franc succès, car elle était considérée par les intellectuels de l'époque comme l'une des plus claires manifestations de l'esprit universel et cosmopolite propre au siècle des Lumières (GARCÍA HURTADO, 1999, p. 38).

Pendant cette période, les traductions n'ont cessé de susciter l'intérêt des Espagnols, à en croire le nombre élevé des ouvrages traduits tout au long du siècle. Grâce à plusieurs études contemporaines, nous pouvons parler de 2117 éditions d'œuvres traduites. Ces chiffres nous aident à comprendre la véritable colonisation linguistique et culturelle que connut le monde des lettres hispaniques de l'époque ${ }^{2}$.

Les traductions dans l'Espagne des Lumières jouissaient d'une telle popularité que, vers la fin du siècle, José Vargas Ponce déclara avec insistance que l'Espagne était devenue une nation de traducteurs, et il forgea un nouveau terme qui devint alors célèbre: «la traductomanie » (VARGAS PONCE, 1793, p. 179). De fait, le développement de l'univers de la traduction favorisa l'arrivée, tout au long du siècle, d'éléments exotiques dans la langue espagnole. Malgré l'opposition d'un groupe de savants liés à l'Académie, fidèles à des principes de pureté de la langue, l'univers de la traduction occupa une place de choix dans la vie culturelle hispanique du temps des Lumières.

L'arrivée des traductions dans le pays, ainsi que leur succès, incita rapidement les traducteurs espagnols à façonner une image du bon traducteur ; celle de quelqu'un capable d'unir, avec une habileté et une intelligence considérables, la langue source et la langue cible, grâce à la connaissance des langues et des coutumes du pays d'origine (VÁSQUEZ-AYORA, 1977, p. 11).

C'est précisément à ce sujet que s'exprima Fernando Guilleman, célèbre traducteur espagnol des Lumières, lorsqu'il traduisit en 1788 Les Veillées du château ou Cours de morale à l'usage des enfants (1784), une œuvre de la célèbre pédagogue des Lumières françaises, Stéphanie Félicité de Genlis :

Para traducir una obra, mayormente si tiene mérito, no basta entender y traducir bien el idioma, ni tampoco bastan ni sirven de mucho los diccionarios,

\footnotetext{
${ }^{2}$ Aujourd'hui, grâce au vaste travail réalisé par Francisco Aguilar Piñal, nous savons que 23,47 \% des traductions, par rapport à la production d'un siècle complet, furent réalisées entre 1780 et 1789 . Pour plus d'informations, on se reportera à son travail intitulé : «Índice de las poesías publicadas en los periódicos españoles del siglo XVIII».
} 
recurso muy débil e imperfecto por su misma naturaleza. Es preciso para emprender este trabajo con alguna esperanza de feliz éxito, haber estudiado el espíritu de la lengua en los mismos que la hablan y haber leído con reflexión muchos libros de todas clases, porque no se usan en todas las obras de las mismas voces, frases ni estilo. El político tiene su momento de expresarse; el orador el suyo; el cómico otro muy diverso; el autor de novelas (si hace lo que debe) se ha de ceñir a un estilo puro, pero familiar y vivo, que es el propio de una conversación o de un dialogo. Es preciso también en el traductor bastante conocimiento de los usos y costumbres de la nación en cuyo idioma está el original; pues sin esto tropezará mil veces en la inteligencia y verdadero sentido de muchas frases. ${ }^{3}$ (GUILLEMAN, 1788, p. 2).

La pratique de la traduction restait donc soumise à la spécificité de chaque œuvre originale, ce qui avait d'autant plus d'importance dans les traductions littéraires en particulier. En effet, la plupart des traducteurs de l'époque réalisèrent leurs propres adaptations, portés par la conviction qu'ils contribuaient au progrès de la nation en mettant à la disposition de la société espagnole les plus grandes découvertes culturelles et scientifiques des autres pays européens (GARCÍA GARROSA, 2012, p. 94). S'impose ainsi l'idée selon laquelle, grâce à ses divers traducteurs, l'Espagne s'attache à combler, en très peu de temps, les lacunes existantes dans les domaines sociaux et culturels, par l'entremise de traductions qui deviennent, tout au long du siècle, un véritable service de bien public.

L’objectif pédagogique, qui fut très souvent invoqué au XVIII ${ }^{\mathrm{e}}$ siècle, fit la part belle à la notion novatrice d'utilité, qui guidait la pratique de la traduction dans quasiment tous les genres - et notamment dans toutes les œuvres destinées, entre autres, à l'instruction de la jeunesse. Les débats éducatifs qui avaient lieu en France circulaient aussi, d'ailleurs, dans le milieu intellectuel espagnol, grâce aux textes originaux qui traversaient les frontières, mais aussi, et surtout, grâce aux différentes traductions publiées. Des traductions qui, d'une certaine

\footnotetext{
${ }^{3}$ Prologue du traducteur : « Pour traduire une œuvre, et d'autant plus si celle-ci est de qualité, il ne suffit point de comprendre et de bien traduire la langue, pas plus que les dictionnaires, cette ressource si faible et imparfaite par sa nature même, ne servent à grand-chose. Pour entreprendre cette tâche en espérant qu'elle soit couronnée de succès, il faut avoir étudié l'esprit de la langue chez ceux-là même qui la parlent, et avoir lu avec intelligence beaucoup de livres de toutes sortes, car l'on n'use pas des mêmes tournures, phrases, ou styles, dans toutes les œuvres. L'homme politique a sa manière de s'exprimer ; l'orateur a la sienne ; le comique en a une autre encore, bien différente ; l'auteur de romans (s'il sait y faire) doit s'astreindre à un style pur, mais familier et vif, qui est le propre d'une conversation ou d'un dialogue. Chez le traducteur également, il faut trouver une connaissance suffisante des us et coutumes, ainsi que de la langue, de la nation où est rédigé l'original; sans cela, celui-ci achoppera mille fois sur l'intelligence et le véritable sens des phrases. » (N.T.)
} 
manière, servirent de relais, tout au long du siècle, à une importante production de traités d'éducation à caractère théorique. Ainsi, puisque l'éducation constitue l'un des principaux axes de la politique des Lumières, les livres destinés aux enfants deviennent alors un outil très efficace pour servir la transmission des connaissances et des valeurs dominantes. Par conséquent, cette motivation didactique fut illustrée par l'importance du nombre d'œuvres françaises ${ }^{4}$ traduites, des œuvres où le divertissement était précisément associé à des contenus instructifs pour faire l'éducation des jeunes filles espagnoles. (MÉRIMÉE, 1936, p. 57).

Par ailleurs, la recherche la plus contemporaine commence à accorder une attention accrue au rôle que jouèrent les femmes dans l'histoire de la traduction espagnole du XVIII ${ }^{\mathrm{e}}$ siècle. Avec l'arrivée des œuvres les plus fondatrices des Lumières européennes, l'univers féminin traditionnel connut un bouleversement indéniable. Le goût pour la lecture et les langues, qui venait d'une grande majorité de femmes appartenant à l'aristocratie et à la bourgeoisie la plus fortunée, ou bien dans d'autres cas, le simple désir de suivre les modes de l'époque, favorisa l'arrivée de nouvelles auteures dans ce que le spécialiste Emilio Palacios qualifie de «jungle des traductions » (2002, p. 91). Les traductions devinrent donc pour les femmes une voie modeste leur permettant d'avoir accès à la culture et aux œuvres les plus remarquées, et parfois également, aux écrivains étrangers les plus audacieux de leur temps.

Les femmes espagnoles traduisirent des œuvres de tout genre : récits de voyages, traités d'histoire, essais philosophiques ou mathématiques et, surtout, des œuvres littéraires (poésie, théâtre, roman) ; cependant leur principal intérêt portait sur les ouvrages à caractère pédagogique, dont elles devinrent des traductrices, mais aussi des créatrices. Ainsi, le métier ou la pratique de traducteur permit à bien des auteurs espagnols, et plus précisément à bien des auteures, d'avoir accès aux ouvrages à succès, fort appréciés à l'étranger. Ana Muñoz, María Jacoba Castilla, María Romero Masegosa, Antonia de Río y Arnedo, Cayetana de la Cerda et tant d'autres se feront tour à tour traductrices et écrivaines, et donneront ainsi une couleur féminine au mouvement d'émancipation et d'éducation de la femme hispanique en particulier, et à l'Espagne des Lumières en général.

Or nous disposons de peu d'information concernant l'éducation ou la formation de ces nouvelles créatrices ; leur bagage était évidemment beaucoup plus sommaire que celui de leurs

\footnotetext{
${ }^{4}$ Il faut souligner la domination incontestée de la langue française, dans la quasi-totalité des traductions. Le français fut la langue source de plus de la moitié des textes traduits en espagnol ; il est suivi de près par l'italien, le latin, l'anglais et le portugais. Pour plus d'informations, on se reportera au travail de Paul Mérimée, «L'influence française en Espagne au XVIII siècle ».
} 


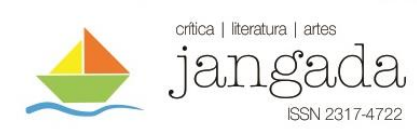

collègues masculins. Toutefois, il est évident que pour l'ensemble de ce groupe d'intellectuelles audacieuses, la traduction devint un tremplin parfait pour servir leur but et faire une incursion timide dans l'univers des lettres, qui était, jusqu'alors, l'apanage des hommes. Grâce à leur dévouement notoire, des productions plus que remarquables sont parvenues jusqu'à nous ; il faut préciser au demeurant que ces textes-là ont joué un rôle fondamental dans les aspirations d'autres femmes, en matière de littérature et de traduction (BOLUFER PERUGA, 1995, p. 42).

D'ailleurs, certaines traductions concoururent à ce que puisse s'élaborer un corpus de textes en espagnol, au sujet de l'éducation des femmes au XVIII ${ }^{\mathrm{e}}$ siècle. Cela contribua à configurer toute une pensée féministe, ou du moins réformatrice, en lien avec la pensée sur l'éducation des femmes et sur leur rôle en société, qui sut se répandre grâce à la diffusion de ces textes dans les salons et dans la presse.

En définitive, dans cette introduction portant sur la situation de la traduction et notamment de sa modalité féminine dans l'Espagne du XVIII ${ }^{\mathrm{e}}$ siècle, nous avons pu comprendre de quelle manière les traductions ont pu représenter une porte ouverte sur les productions étrangères et sur le progrès. Un progrès connu des sociétés voisines mais ignoré en Espagne depuis l'instauration d'une dictature de la terreur inquisitoriale (MONTERO PIZARRO, 2005, p. 11). Cependant, malgré cette période d'omniprésence religieuse, l'important travail de diffusion de certaines de ces œuvres et de ces traductions, mené à bien par la presse espagnole, devint un facteur essentiel à la notoriété qu'acquirent ces auteures françaises dans l'Espagne des Lumières.

Nous évoquerons, parmi ces écrivaines, Louise d'Épinay, Anne-Thérèse de Marguenat de Courcelles, devenue par son mariage madame de Lambert, ou Marie de Rabutin-Chantal, plus connue sous le nom de marquise de Sévigné, pour leur notoriété dans le domaine culturel des Lumières espagnoles. Ainsi, l'analyse des premières versions en langue castillane nous permettra de donner un aperçu des divers travaux de traduction que les lecteurs hispaniques eurent l'occasion de lire à l'époque. Des traductions parfois incomplètes, incertaines ou encore imprécises qui, directement ou indirectement, marquèrent la fortune littéraire de ces auteures dans l'Espagne des Lumières.

LOUISE-FLORENCE-PÉTRONILLE TARDIEU D'ESCLAVELLES : MADAME D'ÉPINAY ET SA FORTUNE LITTÉRAIRE DANS L'ESPAGNE DES LUMIÈRES. 
Chez «la Ilustrada » espagnole Ana Muñoz, Louise d'Épinay trouva la promotrice idéale de ses célèbres Conversations d'Émilie, publiées en 1774 et très vite reconnues en France. Cette œuvre fut traduite pour la première fois en langue castillane en 1797.

La popularité de cette production traversa rapidement les frontières franco-espagnoles. Ce nouvel ouvrage pédagogique était présenté comme une méthode inédite pour mieux orienter les destins des futures mères. Il faut souligner que jusqu'alors, l'Émile (1762) de Jean-Jacques Rousseau constituait une source de règles dans le domaine de la pédagogie. Cependant, Louise d'Épinay, bien loin des préceptes rousseauistes de docilité et de modestie, proposa aux femmes d'autres moyens pour atteindre le bonheur, aux antipodes des principes traditionnels d'obéissance et de soumission aux figures masculines.

La publication d'une méthode aussi différente de celle de Rousseau octroya une grande renommée à cette œuvre et à son auteure. Grâce à cette popularité transfrontalière, une première annonce timide parut dans le journal la Gaceta de Madrid, qui faisait référence à un ouvrage inconnu jusqu'alors pour les lecteurs hispaniques, et ce, avant même l'arrivée dans les librairies de la première version espagnole de l'ouvrage.

Les Conversations d'Émilie de Louise d'Épinay furent également recensées dans les journaux Mercurio histórico et Diario de Valencia, qui y virent un texte témoignant d'une bonne éducation chrétienne, morale et politique grâce à des réflexions simples, sans artifices, promises à un bon accueil chez les plus jeunes lecteurs de l'époque (Mercurio histórico, 1789, p. 111). Une autre preuve de la popularité et de la bonne réception de cette œuvre en Espagne fut la publication, en 1797 et sans mention du nom de l'auteure, de deux des conversations entre Émilie et sa mère, dans le journal littéraire madrilène La Miscelánea instructiva y curiosa. Dans le tableau comparatif qui suit, nous présentons les premières lignes de cette traduction que les Espagnols purent lire dans les pages du journal, puis la version originale française correspondante.

\begin{tabular}{|l|l|}
\hline \multicolumn{1}{|c|}{ Les Conversations d'Émilie (1774) } & $\begin{array}{l}\text { Conversación de Emilia con su Madre } \\
\text { (Extrait de la traduction, publié en 1798) }\end{array}$ \\
\hline $\begin{array}{l}\text { Émilie : Maman, pourquoi suis-je au monde ? } \\
\text { La Mère : Pour cette question, c'est à moi à vous } \\
\text { la faire. }\end{array}$ & $\begin{array}{l}\text { Emilia: Mamá, ¿Para qué estoy yo en el } \\
\text { mundo? } \\
\text { La Madre: Vaya: dímelo tú a mí. }\end{array}$ \\
\hline
\end{tabular}




Émilie : Ah, je m'en souviens maman...Faut-il
aussi que j'y sois utile ?
La Mère : Si vous ne l'êtes pas encore, il faut du
moins vous mettre en état de le devenir un jour.
Émilie : Maman, je me promène, je saute, je bois,
je mange, je ris, j'étudie, je cause avec vous tant
que je veux : tout cela est-il bien utile aux autres?

La Mère : Pour pouvoir l'être un jour, il faut avoir acquis avec la maturité de l'âge la force et la santé ; et pour les acquérir, il faut boire, manger, dormir, sauter, rire. Il me semble que vous remplissez avec distinction tous ces devoirs. Émilie : J'en suis bien aise.

La Mère : À mesure que vous grandirez, vos occupations et vos obligations changeront; vous deviendrez véritablement utile, au lieu d'être à charge aux autres.

Émilie : D'être à charge aux autres ! Est-ce que je suis à charge ?

La Mère : Comment! Vous avez cinq ans passés, et vous ne l'avez pas encore remarqué ? Un enfant est dans la dépendance de tout le monde par sa faiblesse : quand on a à tout instant besoin des autres, on court sûrement le risque d'être souvent à charge.

Émilie : Est-ce que je vous suis à charge, maman?

La Mère : La nature a accordé aux parents un préservatif contre cet inconvénient.

Émilie : Quel préservatif?

La Mère : La tendresse qu'elle a mise dans leur cœur, et qui change en jouissances les soins les plus pénibles, les plus assidus, dont leurs enfants ont besoin. Une mère ne connaît pas de plus doux,
Emilia: ¿Si yo no lo sé?

La Madre: ¿Qué es lo que haces todo el día?

Emilia: Me paseo, doy lección, salto, bebo, como, río y hablo con Vd. Cuando estoy buena.

La Madre: Bien pues hasta ahora para eso estás en el mundo, para beber, comer, dormir, reír, saltar, crecer, robustecerte e instruirte.

Esto es lo que tienes que hacer, conforme vayas creciendo, se mudarán tus ocupaciones y tus obligaciones. En lugar de estar en el mundo para saltar y bailar y servir de carga a los demás, estarás para trabajar, para ser útil, para cumplir otras obligaciones y disfrutar de otras diversiones.

Emilia: ¿Servir de carga a los demás? ¿Pues que yo sirvo de carga?

La Madre: Sin duda: porque eres una niña.

Emilia: Pero una niña es una persona

La Madre: Una niña, es una niña que con el tiempo vendrá a ser una persona.

Emilia: ¿Pero, qué quiere Vd. Decir con que yo no soy más que y una niña?

La Madre: ¿Con que ya tienes cinco años y todavía no lo has conocido? Averígualo tú por ti misma.

Emilia: Mama yo no lo comprendo

La Madre: Pues una niña es una criatura débil, dependiente de todos, inocente e ignorante, atolondrada, importuna e indiscreta.

Emilia: ¿Con que yo tengo todas esas faltas?

La Madre: Todas son faltas de tu edad. Ya ves que una niña todo se lo debe al cariño de sus 


\begin{tabular}{|c|c|}
\hline $\begin{array}{l}\text { de plus grand plaisir que de s'occuper de sa fille. } \\
\text { Ignorance, étourderie, indiscrétion, importunité, } \\
\text { tout est supporté par la tendresse maternelle. } \\
\text { Émilie : Quoi, j'ai tous ces défauts? } \\
\text { La Mère : Ce sont ceux de votre âge. } \\
\qquad[\ldots] \\
\text { Émilie : Je n'avais jamais pensé à tout cela. Mais } \\
\text { aussi je ne suis pas bien méchante, je crois. } \\
\text { La Mère : Quand on dépend si fort des autres, } \\
\text { vous voyez combien on a des raisons d'être } \\
\text { douce, polie, reconnaissante. }\end{array}$ & $\begin{array}{l}\text { padres, y que no es sino una carga insoportable } \\
\text { para los demás. } \\
\text { Emilia: Verdad es, Mamá que yo sería digna } \\
\text { de lástima, ni me podría levantar ni acostar ni } \\
\text { hacer nada sola. } \\
\text { La Madre: Ya ves que cuando se necesita de } \\
\text { todos, es necesario ser afable, suave y } \\
\text { agradecida: corregir el mal humor, } \\
\text { aprovecharse de las lecciones y consejos y } \\
\text { conocer que cuando te corrigen es porque te } \\
\text { quieren porque desean que seas amable. }\end{array}$ \\
\hline
\end{tabular}

Tableau 1. Les Conversations d'Émilie

Les ressemblances entre les deux versions s'imposent par leur évidence et nous font d'abord remarquer que le degré d'équivalence sémantique des deux textes est plutôt important. Cependant, et malgré une activité interdiscursive manifeste, un certain changement de style est souligné dans la traduction espagnole, ce qui oblige la traductrice à supprimer certains passages d'un côté, et à amplifier quelques phrases de l'autre. Toutefois, ce ne sont pas là des techniques innovantes pour les traducteurs espagnols de l'époque, puisque la plupart des traductions des Lumières partageaient le même genre de méthodes et d'approches. Ce n'est pas tant pour la démarche dans laquelle s'inscrit cette traduction que nous avons reproduit ces lignes que pour leur publication inattendue, et anonyme de surcroît, dans l'un des journaux espagnols les plus célèbres de l'époque.

En dépit de cela, les éloges sur les Conversations d'Émilie, loin de s'étioler, continuèrent à occuper de plus en plus de place dans d'autres journaux de l'époque. Citons, à titre d'exemple, la publication du mardi 4 juillet 1797 de la Gaceta de Madrid, où nous pouvons lire :

Conversaciones de Emilia; escritas en francés por Madame Live de Épinay y para instrucción de su familia, y proporcionar a los que tienen semejante cuidado un medio fácil y eficaz de cumplir tan importante obligación, y procurar a sus hijos y domésticos una crianza cristiana y política. Desempeña ese importante objeto con cuentos ingeniosos, dichos oportunos y sencillas 


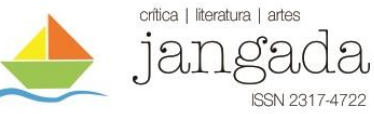

reflexiones propias para entretener sin fastidios los niños y fijar en su alma las sólidas máximas que contienen, e inspirar el conocimiento del corazón humano, tan preciso para vivir en el mundo. Como obra la más a propósito y acomodada para este fin, se imprimió repetidas veces en Francia, se prefirió a otras por orden de Luis XVI para las escuelas y colegios de ambos sexos, y se tradujo en varias lenguas, y ahora en la nuestra sobre la $5^{\mathrm{a}}$ edición por doña Ana Muñoz, para utilidad principalmente de las madres de familia. ${ }^{5}$ (Gaceta de Madrid, 1797, p. 960).

La fortune littéraire de cette écrivaine française ne fut pas révélée par les seuls journaux de l'époque. Ainsi, Josefa Amar y Borbón, Josefa de Jovellanos ou Rita Caveda Solares sont de ces intellectuelles espagnoles qui firent l'éloge de cette œuvre et de sa brillante auteure dans les différents prologues de leurs propres écrits (CAPEL MARTÍNEZ, 2010, s/p).

Du fait de la popularité de l'auteure française et de son œuvre, il n'est pas surprenant que la traduction publiée par Ana Muñoz, une année à peine après la diffusion des lignes citées ci-dessus, ait été si bien acceptée par les différents intellectuels de l'époque. Cependant, nous ne devons pas oublier que du fait de l'omniprésence de l'Église Catholique dans l'Espagne des Lumières, la grande majorité des traductions publiées tout au long du siècle eut à s'adapter à la morale religieuse du moment. Un fort protectionnisme, instauré et tenu par l'institution religieuse de l'Inquisition, entrava la publication de toute traduction dont les propos idéologiques et philosophiques pouvaient aller directement à l'encontre de la morale espagnole imposée à l'époque ${ }^{6}$. Ainsi, nous ne pouvons pas parler de traductions orthodoxes pour les traductions publiées pendant ce siècle, mais plutôt d'adaptations d'œuvres au contexte particulier des Lumières espagnoles.

\footnotetext{
${ }^{5}$ Publication de la Gaceta de Madrid du mardi 4 juillet 1797 : «Les Conversations d'Émilie ; écrites en français par Madame Live d'Épinay pour l'éducation de sa famille, et dans le but de fournir, pour ceux qui ont un souci semblable, un moyen facile et efficace d'accomplir une obligation si importante, et de procurer à leurs enfants et domestiques une instruction chrétienne et politique. Le livre atteint cet objectif primordial grâce à des contes courts, appropriés, ingénieux, et à des dictons simples qui viennent à point nommé, faits pour amuser les enfants sans susciter d'ennui, et pour leur inculquer les maximes solides qu'ils contiennent, et inspirer la connaissance de l'esprit humain, si précieuse pour vivre dans le monde. S'agissant d'une œuvre qui tombe à propos et qui est des plus adéquates pour parvenir à cette fin, elle fut imprimée à plusieurs reprises en France, et préférée à d'autres sur l'ordre de Louis XVI pour être étudiée dans les écoles et les collèges des deux sexes ; elle fut également traduite dans d'autres langues, et maintenant dans la nôtre à partir de la $5^{\mathrm{e}}$ édition, grâce à Ana Muñoz, principalement pour servir les mères de famille ». (N.T.)

${ }^{6}$ Il faudra quasiment attendre la fin du XIX ${ }^{\mathrm{e}}$ siècle pour trouver les premières traductions fidèles, en langue castillane, de certaines des œuvres les plus notoires des Lumières françaises.
} 
Les différences entre les deux versions sont visibles d'emblée. Les premières pages que nous lisons dans l'œuvre originale comprennent une adresse à « Catherine II, Impératrice et Autocratrice de toutes les Russies ». Il s'agit d'une épître où Louise d'Épinay salue la bienveillance et l'intérêt que l'impératrice porte à son œuvre, épître qui n'a jamais été traduite en espagnol (pas même dans les éditions espagnoles les plus récentes, où nous n'avons trouvé aucune trace de cette lettre). Nous pouvons imaginer que la femme de lettres espagnole considéra que cette information était superflue pour ses futurs lecteurs.

Comme nous l'avons remarqué auparavant, la plupart des traductions-adaptations des Lumières qui nous sont parvenues mènent à confirmer l'hypothèse selon laquelle la plupart des versions furent publiées à des fins d'instruction de la société espagnole. Dans la plupart des cas, elles comptèrent, parmi leurs lecteurs, des novices sans connaissance des lettres françaises ni même des événements qui s'étaient produits loin des frontières espagnoles. De ce fait, les questionnements de la plus grande partie des traducteurs hispaniques de l'époque ont dû reposer sur cette interrogation : à quoi bon traduire certains passages, s'ils n'ont aucun objectif pédagogique et ne font que contribuer à brouiller la compréhension finale du texte ?

Après l'épître introductive, l'œuvre originale se poursuit avec une lettre de Madame d'Épinay elle-même, qui est adressée à l'éditeur de la première édition de son œuvre (1774). Elle y défend son travail face aux éventuelles critiques, en manifestant qu'elle n'avait pas prétendu là proposer un nouveau traité d'éducation, et qu'elle n'avait eu pour intention que de composer un traité pour former l'esprit d'un enfant et lui inspirer des sentiments vertueux et honnêtes. (ÉPINAY, 1788, p. 3). Ces justifications personnelles de l'auteure, suivies de l'avertissement de l'éditeur sur la seconde édition rédigée en 1781, et l'approbation et l'avertissement de l'éditeur dévoilées dans les éditions postérieures, celles de 1782 et 1788 , seront également absentes de la version hispanique.

Malgré ces omissions évidentes, la traductrice espagnole s'emploie, tout au long des premières pages de sa version, à rédiger un bref prologue où elle s'adresse directement à ses futurs lecteurs, en faisant une plaidoirie, sur un ton de proximité et de familiarité, à propos de l'importance de l'éducation des enfants pour le bon développement du pays.

Si consideras, amado Lector, que la instrucción, así intelectual como moral de la juventud, es el carácter de toda República bien ordenada e indispensable en el gobierno doméstico de una familia; no te hará fuerza hayan sido tan 
repetidas las impresiones de estas Conversaciones de Emilia, que para beneficio común supo disponer con tanto acierto Madama de Live de Épinay. ${ }^{7}$ (ÉPINAY, 1788, p. 3).

Ana Muñoz inclut également dans son prologue une dure critique envers les institutrices de l'époque, en désignant « el mal humor y desabrida superioridad de que las hace revestir el título de Maestra $»^{8}(1788$, p. 5) comme étant les responsables directs de la mauvaise éducation des jeunes demoiselles hispaniques. C'est la raison pour laquelle la traductrice décida d'entreprendre la version d'une œuvre qui était si importante pour elle, et si nécessaire à ce que la nation espagnole «no carezca de la mucha utilidad que de su lectura se puede esperar con ahorro de muchos gastos y sinsabores $»^{9}(1788$, p. 5). À la suite de ce prologue, Ana Muñoz commence sa traduction.

En confrontant les deux textes, nous pouvons mettre en évidence les premières différences. Ici encore, nous avons l'impression que lorsque Ana Muñoz entreprit ce travail de traduction, elle se trouva obligée, à plusieurs reprises, d'adapter et de simplifier son texte pour qu'il s'ajuste à la mentalité et au langage usuel de ses lecteurs.

\begin{tabular}{|l|l|}
\hline \multicolumn{1}{|c|}{$\begin{array}{c}\text { Les Conversations d'Émilie } \\
\text { (1774) }\end{array}$} & \multicolumn{1}{c|}{\begin{tabular}{c}
\multicolumn{1}{c|}{ Las Conversaciones de Emilia } \\
(1797)
\end{tabular}} \\
\hline Émilie : Voilà Rosette qui mange ma robe ! & $\begin{array}{l}\text { Emilia: Ay madre, vea usted cómo la perrilla } \\
\text { me come los vestidos. }\end{array}$ \\
\hline $\begin{array}{l}\text { La Mère : La nature a accordé aux parents un } \\
\text { préservatif contre cet inconvénient. }\end{array}$ & $\begin{array}{l}\text { La Madre: Pues te engañas, porque con un } \\
\text { bofetón se te puede echar al suelo, y aún } \\
\text { matarte. }\end{array}$ \\
\hline $\begin{array}{l}\text { La Mère : La tendresse qu'elle a mise dans leur } \\
\text { cœur et qui change en jouissances les soins les } \\
\text { plus pénibles, les plus assidus, dont leurs enfants } \\
\text { ont besoin. Une mère ne connaît pas de plus doux, }\end{array}$ & $\begin{array}{l}\text { La Madre: No, porque tu ignorancia y poca } \\
\text { reflexión no te permite prever ni evitar el } \\
\text { peligro, y tu debilidad el librarte de él. } \\
\text { Necesitas continuamente de una persona que te }\end{array}$ \\
\hline
\end{tabular}

\footnotetext{
${ }^{7}$ « Cher Lecteur, si vous considèrez que l'instruction de la jeunesse, intellectuelle et morale tout aussi bien, est le principe de toute République bien ordonnée, et est indispensable au gouvernement domestique d'une famille, vous ne serez pas surpris de trouver des rééditions fréquentes des Conversations d'Émilie, que les bons soins de Madame de Live d'Épinay ont su disposer pour le bien de tous ». (N.T.)

8 «La mauvaise humeur et la supériorité hargneuse dont elles font preuve dans leur rôle de maîtresse ». (N.T.)

${ }^{9}$ « [La nation espagnole] ne se passe pas de l'utilité énorme que l'on peut tirer de sa lecture, en s'épargnant ainsi bien des ennuis et des déboires ». (N.T.)
} 


\begin{tabular}{|c|c|}
\hline $\begin{array}{l}\text { de plus grand plaisir que de s'occuper de sa fille. } \\
\text { Ignorance, étourderie, indiscrétion, importunité, } \\
\text { tout est supporté par la tendresse maternelle. }\end{array}$ & $\begin{array}{l}\text { guarde, y nadie tiene interés en ello porque tú } \\
\text { ¿con qué puedes recompensar? Si alguno le } \\
\text { tiene, será por los respetos que se te deben, por } \\
\text { tu afabilidad y docilidad, con cuyas } \\
\text { circunstancias te podrás prometer continuarán } \\
\text { sirviéndote; pero si tienes mal genio, si } \\
\text { respondes con aspereza, y la bondad de tu } \\
\text { corazón no te manifiesta el reconocimiento y } \\
\text { obligación que tienes a todos los que cuidan de } \\
\text { ti, debilitará prontamente la compasión natural } \\
\text { que inspira tu eterna edad; te abandonarán y en } \\
\text { esta situación, ¿qué será de ti? }\end{array}$ \\
\hline $\begin{array}{l}\text { La Mère : Eh bien, ma fille, un mauvais caractère } \\
\text { rendait malheureusement Julie insensible aux } \\
\text { conseils de sa mère qui, longtemps incertaine } \\
\text { entre sa tendresse et sa raison, mais voyant sa fille } \\
\text { incorrigible, fut enfin obligée de la mettre au } \\
\text { couvent, l'exemple même de son frère n'ayant fait } \\
\text { aucune impression sur elle. Cette retraite n'opéra } \\
\text { aucun changement avantageux dans son caractère. } \\
\text { Bientôt elle manqua un mariage considérable que } \\
\text { sa beauté et ses agréments extérieurs lui auraient } \\
\text { procuré, parce que les informations qu'on prit au } \\
\text { couvent à son sujet, lui furent entièrement } \\
\text { défavorables. (ÉPINAY, 1774, p. 23). }\end{array}$ & $\begin{array}{l}\text { La Madre: Aunque Julia observaba que su } \\
\text { hermano tenía un gran respeto y una entera } \\
\text { confianza en su madre, y jamás se acercaba a } \\
\text { ella, sin darla primero señales de esta } \\
\text { obligación y que su mayor temor eta } \\
\text { desagradarla; de nada la sirvió este ejemplo: } \\
\text { porque ella no solo era ingrata para su madre, } \\
\text { sino también de genio muy áspero, caprichuda, } \\
\text { perezosa, muy pronta en enfadarse y siempre } \\
\text { echaba a otros la culpa que ella tenía; por lo } \\
\text { que viendo la madre que era incorregible, se } \\
\text { vio precisada a ponerle en un Convento. Y } \\
\text { aunque después se la proporcionaba un gran } \\
\text { matrimonio, luego se desvaneció, porque los } \\
\text { informes que dieron en el Convento fueron } \\
\text { para ella tan poco favorables que la } \\
\text { despreciaron sin hacer mérito de su hermosura. } \\
\text { (ÉPINAY, 1797, p. 16). }\end{array}$ \\
\hline
\end{tabular}

Tableau 2. Les Conversations d'Émilie

Dans cette version, il nous faut souligner qu'à la différence d'autres traductions publiées au cours du XVIII ${ }^{\mathrm{e}}$ siècle, Ana Muñoz ne traduisit ni tous les prénoms ni les références françaises présentes dans la version originale. Même si, dans certains exemples soulignés Jangada | nr. 12, jul/dez, 2018 | ISSN 2317-4722 


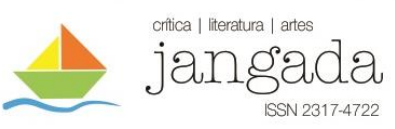

auparavant, nous pouvons constater une « hispanisation » ou tout simplement la suppression de certains prénoms, nous discernons aussi quelques mots qui appartiennent à la version originale. «Pour hériter de sa terre de Champorcé-le-Vicomte » est traduit par « escogido por heredero del vizcondado de Champorcé »; de même, « de m'épargner les chagrins de me transporter à Paris » est traduit par «y ahorrarme el trabajo de volver a París ». Ainsi, contrairement à d'autres traductions publiées pendant cette même période, où les références aux villes françaises étaient bannies, Ana Muñoz livre une version plutôt fidèle, tout en sachant s'adapter aux mentalités et aux connaissances espagnoles. Cette adaptation a permis de préserver l'essence originale du texte français, au profit d'une lecture de cette version qui soit en fin de compte plus compréhensible.

Malgré ces progrès concernant l'art de la traduction, la fidélité est également un point discutable dans la nouvelle version. Si Ana Muñoz demeure assez proche des thèmes évoqués par Louise d'Épinay, tout comme une grande majorité des traducteurs des Lumières, elle adapte et transforme sa version par le biais d'expressions et de tournures de phrases propres à la langue orale espagnole. En définitive, la réalisation d'une traduction moins littérale permet ici la transposition des idées, afin d'arriver à un résultat plus naturel et plus compréhensible pour les lecteurs de l'époque.

Nous pouvons nous interroger à présent sur les raisons qui menèrent le Saint-Office à donner un avis favorable sur une traduction tellement «francisée ». La censure espagnole au XVIII ${ }^{e}$ siècle est un sujet trop connu pour qu'on y revienne dans ces pages ; cependant, nous ne devons pas oublier que l'index inquisitorial publié en 1747 comprenait un ratio de livres en français beaucoup plus important que dans l'index précédent. Cela était dû, précisément, à cette dictature religieuse omniprésente, qui condamnait quasiment par principe la plupart des romans, à cause de leurs origines françaises (VÁSQUEZ, 1991, p. 36). Le travail de traduction d'Ana Muñoz fut publié en 1797, sous le règne de Charles IV. Nous savons que ce monarque instaura, par rapport aux périodes précédentes, un climat de liberté et de permissivité assez relatives, où la terreur nourrie par l'Inquisition pendant les années précédentes diminua remarquablement. Cette nouvelle réalité favorisa donc la baisse considérable de sanctions, qui se firent plus rares et moins sévères qu'auparavant.

Andrés Muriel, historien de Charles IV et témoin direct de cette nouvelle réalité sociale et culturelle de l'Espagne des Lumières, affirmait : 
¿La Inquisición? Su antiguo poder no existía ya: la autoridad horrible que este Tribunal sanguinario había ejercido en otros tiempos quedaba reducida a muy estrechos límites, pues el Santo Oficio había venido a parar a ser una especie de comisión para la censura de libros; no más, y aún para conservar esta existencia tenía necesidad de ser sufrida y tolerante. ${ }^{10}$ (MURIEL, 1963-64, p. 270).

Toutes ces évidences répondent à nos questions concernant l'indulgence avec laquelle cette traduction fut traitée. En outre, Ana Muñoz décida de traduire également tous les passages concernant l'éducation limitée des jeunes demoiselles - sujet polémique s'il en est dans l'Espagne de l'époque.

No se acostumbraba a instruir a las niñas: se las enseñaba muy superficialmente los principios de la religión [...] Con eso, un poco de historia, y de geografía, pero sin gusto alguno [...]. Jamás nos enseñaban cosas razonables; y tocante a la ciencia la creían muy excusada en nosotras, y huían con el mayor cuidado de toda especie de instrucción. ${ }^{11}$ (ÉPINAY, 1774, p. 329).

Nous pouvons à ce propos rappeler le combat épistolaire initié par l'intellectuelle hispanique Cayetana de la Cerda y Vera, à la suite du refus par le Saint-Office de publier sa traduction de Les Américaines, ou la Preuve de la religion chrétienne par les lumières naturelles $^{12}$ (1770) de Marie Leprince de Beaumont. Cet exemple nous aide à déceler chez l'Inquisition une certaine bienveillance à l'égard du travail de traduction d'Ana Muñoz. De toute évidence, la publication d'une version qui défendait si ouvertement l'éducation complète

\footnotetext{
${ }^{10}$ «L'Inquisition? Son ancien pouvoir n'existait déjà plus : l'horrible autorité que ce Tribunal sanguinaire avait exercée jadis fut cantonnée dans d'étroites limites, car le Saint-Office n'était plus qu'une simple commission pour la censure des livres et, pour la seule conservation de ce statut, il devait se montrer résigné et tolérant. » (N.T.)

${ }^{11}$ « Ce n'était pas l'usage de rien apprendre aux filles. On leur enseignait les devoirs de religion, [...] Avec cela un peu d'histoire et de géographie, mais sans aucun attrait [...]. Surtout, on ne nous parlait jamais raison ; et quand la science, on la trouvait très déplacée dans les personnes de notre sexe et l'on évitait avec soin toute espèce d'instruction. » V.O. (ÉPINAY, 1774, pp. 442-443)

${ }^{12}$ María Cayetana de la Cerda y Vera, comtesse de Lalaing, entreprit en 1790, la traduction de l'une des ouvres de la pédagogue française Marie Leprince de Beaumont, Les Américaines, ou la Preuve de la religion chrétienne par les lumières naturelles (1770). La traductrice, cherchant à rester fidèle aux propos présents dans l'œuvre de l'auteure française, reçut un avis défavorable de la part du Saint-Office qui décida d'interdire la publication de la traduction de l'ouvrage, sous prétexte qu'il était écrit et traduit par une plume féminine et qu'il portait atteinte à la foi chrétienne. Pour plus d'informations, se reporter au vaste travail de Mónica Bolufer (2002): «Pedagogía y moral en el siglo de las Luces: las escritoras francesas y su recepción en España», in Revista de historia moderna, $n^{\circ} 20,251-292$.
} 
des femmes, pendant la période la plus absolutiste du Saint-Office, aurait été censurée ipso facto.

En définitive, Louise d'Épinay, comme nombre de ses contemporaines, devint une auteure renommée en Espagne grâce à une production plaisante, de lecture agréable, et qui, malgré les passages obligés sur l'instruction morale, ne manque pas de vivacité, ni même de quelques traits d'humour. Ce sont là des raisons suffisantes pour penser que cette traduction fit le bonheur de bien des lecteurs de l'époque.

\section{ANNE-THÉRÈSE DE MARGUENAT DE COURCELLES, MADAME DE LAMBERT : UNE PÉDAGOGUE CONTRE LA DICTATURE MASCULINE DES LUMIÈRES.}

Un auteur espagnol disait que le Livre de Don Quichotte avait perdu la Monarchie d'Espagne, parce que le ridicule qu'il a répandu sur la valeur que cette Nation possédait autrefois dans un degré si éminent, en a amolli et énervé le courage. Molière en France, a fait le même désordre par la Comédie des Femmes Savantes. Depuis ce temps-là, on a attaché presque autant de honte au savoir des Femmes, qu'aux vices qui leur sont le plus défendus. (LAMBERT, 1727, p. 160)

Ce passage tiré des Réflexions nouvelles sur les femmes, publiées en 1727, par AnneThérèse de Marguenat de Courcelles, devenue par son mariage madame de Lambert, vient à point nommé pour donner un aperçu de la critique acerbe que cette intellectuelle française brandit contre la ridiculisation des envies intellectuelles féminines, élément si caractéristique des comédies du célèbre dramaturge parisien. « Ne doit-il pas leur suffire de régler tout le mouvement de notre cœur, sans se saisir encore de notre intelligence ? (LAMBERT, 1727, p. 12).

Anne-Thérèse de Lambert devint une fervente défenseure d'une éducation féminine complète, où la raison et les sentiments n'allaient pas l'un sans l'autre. De ce fait, selon cette pédagogue, l'instruction des jeunes demoiselles ne devait pas se limiter à éveiller leur sensibilité ou leur savoir-faire, si nécessaires à la vie sociale ; elle devait aussi proposer une méthode pédagogique grâce à laquelle les femmes puissent apprendre individuellement à comprendre, penser et raisonner, sans recourir à une figure masculine. 


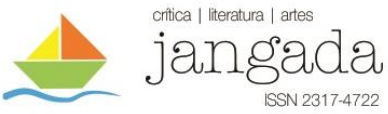

On a dans tous les temps négligé l'éducation des filles [...] comme si les femmes étaient une espèce à part, on les abandonne à elles-mêmes sans secours, sans penser qu'elles composent la moitié du Monde [...] qu'elles font le bonheur ou le malheur des hommes, qui toujours sentent le besoin de les avoir raisonnables. (LAMBERT, 1729, p. 55).

Rapidement, ces propos «féministes », si révoltés et novateurs, traversèrent la frontière franco-espagnole et occupèrent une place de choix dans certains écrits féminins des Lumières espagnoles. Après avoir parcouru le panorama littéraire de l'époque, nous avons pu ressentir la forte influence de cette intellectuelle française dans plusieurs textes hispaniques issus d'une plume féminine.

Con razón se ha considerado siempre la educación como el asunto más grave y más importante. De él depende la felicidad pública y privada [...]. Así cuando mejor fuese la educación, será mayor el número de las personas felices y más grandes las ventajas de aquella república [...]. La educación de las mujeres se considera regularmente materia de poca entidad. El estado, los padres y lo que es más, hasta las mismas mujeres miran con indiferencia el aprender esto o aquello o no aprender nada ¿quién podrá señalar la causa de este descuido tan universal? ${ }^{13}$ (AMAR Y BORBÓN, 1790, pp. 57-59).

Josefa Amar y Borbón, par exemple, s'adonna particulièrement à la lecture des productions d'Anne-Thérèse de Lambert. Les deux écrivaines illustrent l'importance qu'ont les connaissances et le choix des bonnes lectures pour réussir à gagner son indépendance, tellement nécessaire pour les femmes des Lumières. Cette femme de lettres espagnole n'hésita pas à reconnaitre cette forte influence française, si évidente dans sa production littéraire. Soulignons, par exemple, ce passage de son célèbre Discurso sobre la educación física y moral de las mujeres (1790), où elle paraphrase directement certaines lignes de l'Avis d'une mère à sa fille (1728) de l'écrivaine française :

\footnotetext{
13 «À juste titre, l'éducation a toujours été considérée comme le sujet le plus grave et le plus important. Le bonheur public et privé en dépend [...]. Ainsi, plus l'éducation est de qualité, plus le nombre de personnes heureuses augmente et plus les avantages qu'en tire la République sont importants [...]. L'éducation des femmes est régulièrement considérée comme une matière de peu d'envergure. L'Etat, les parents, et jusqu'aux femmes ellesmêmes ne s'occupent guère d'apprendre ceci plutôt que cela, ou que rien du tout ; qui pourrait mettre en évidence cette négligence universelle? » (N.T.)
} 


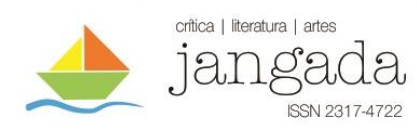

¿Qué mayor ventaja que la de poder hacer un uso saludable del tiempo, prevenir recursos para todas las edades y sucesos de la vida, adquirir nuevas ideas, y estar contento fuera del bullicio de las demás gentes? [...] ¡Qué fortuna es saber vivir consigo mismo, apartarse de sí con violencia, y volver con gusto a encontrarse! Entonces no se apetece el bullicio de las otras gentes. ${ }^{14}$ (AMAR Y BORBÓN, 1790, p. 195).

Amar y Borbón, tout comme Anne-Thérèse de Lambert, venait nourrir la polémique autour de l'éducation féminine, ainsi que les réticences de certains cercles sociaux de l'époque, opposés à cette instruction. Malgré cette situation, à vrai dire peu favorable pour tous ces textes écrits par une plume féminine, la production littéraire de Madame de Lambert fut traduite et publiée pendant le XVIII ${ }^{\mathrm{e}}$ siècle espagnol.

Grâce au travail de traduction conséquent qui fut mené à bien par María Cayetana de la Cerda, comtesse de Lalaing, Madame de Lambert connut un succès timide, en dépit de sa forte influence auprès de quelques intellectuelles hispaniques - et en particulier dans certains cercles plutôt conservateurs. À la différence d'autres auteures françaises des Lumières ${ }^{15}$, cette écrivaine ne jouit pas de la même popularité que ses contemporaines. Cependant, force est de constater que dans son pays d'origine, les productions littéraires de la pédagogue française ne connurent pas non plus une énorme célébrité. Les commentaires, à vrai dire peu flatteurs, publiés dans le journal français le Mercure de France, en 1773, mettent en évidence cette réalité regrettable.

Plusieurs des morceaux qui forment ce recueil méritaient peu les honneurs d'une fréquente réimpression. [...]. À l'exception d'une ou deux lignes, il n'est guère de dissertateur qui ne parlât aussi bien, ou peut-être avec plus justesse que le Diogène de Madame de Lambert, et il n'est pas de personnage

\footnotetext{
14 «Quel plus grand avantage que celui de pouvoir faire un bon usage de son temps, de se prémunir avec des recours pour tous les âges et les événements de la vie, d'acquérir de nouvelles idées, et d'être heureux, en dehors du tumulte des autres gens? Quel bonheur que de savoir vivre avec soi-même, de se quitter soi-même violemment, et de se retrouver avec plaisir ! Le monde bruyant vous est alors moins nécessaire. » (N.T.).

${ }^{15}$ Stéphanie Félicité de Genlis, ou Marie Leprince de Beaumont furent par exemple deux des auteures françaises les plus traduites pendant les Lumières espagnoles. La morale religieuse, plutôt traditionnelle, ainsi que la solennelle morale conventionnelle de leurs modèles pédagogiques, leur langage familier propre au contexte social espagnol, et leurs propos religieux proches des idéologies ecclésiastiques imposées par le tribunal de l'Inquisition, contribuèrent à la popularité des productions littéraires de ces deux pédagogues françaises.
} 


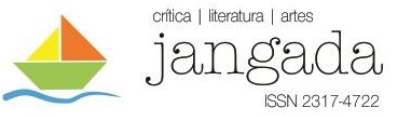

moins héroïque, ou même plus nul que son Alexandre. (Mercure de France, journal littéraire et politique, 1733, p. 449).

De même que chez ses homologues français, la presse espagnole des Lumières, véritable moteur de diffusion de nouvelles voies d'expression ainsi que de divulgation des nouvelles idéologies, ne consacra quasiment aucune page à la production de Madame de Lambert, à la différence d'autres auteures ${ }^{16}$. Les rares traces journalistiques que nous avons retrouvées sont les quelques lignes où la Gaceta de Madrid annonce la publication de la traduction réalisée par la comtesse de Lalaing, et les brèves références incluses dans le Memorial literario :

Obras de la Marquesa de Lambert para la buena educación moral y la política: traducidas nuevamente del francés por Doña María Cayetana de la Cerda y Vera, condesa de Lalaing. Se hallarán en la Imprenta y Librería de Manuel Martín calle de la Cruz. ${ }^{17}$ (Memorial literario, 1781, p. 832).

Nous pouvons à ce titre nous interroger sur les raisons pour lesquelles une œuvre pédagogique féminine comme celle de Madame de Lambert eut à souffrir d'un tel manque de considération dans la péninsule Ibérique. Notre première hypothèse a été de nous intéresser à la qualité du travail de traduction : une mauvaise traduction aurait sans doute pu être tenue pour responsable de ce manque de considération.

Commençons donc notre étude comparative sur les dissemblances pouvant exister entre les deux textes, en français et en espagnol. Si nous revenons aux différentes publications de l'œuvre originale, nous constatons que la première édition des Euvres complètes d'AnneThérèse de Lambert date de 1747. Cependant, cette édition était composée uniquement d'un Abrégé sur la vie de la marquise de Lambert, de l'Avis d'une mère à son fils et de l'Avis d'une mère à sa fille. C'est seulement à partir de la troisième édition, publiée en 1749, que nous pouvons repérer une table des matières aussi longue que celle que l'on trouve traduite dans la

\footnotetext{
${ }^{16}$ Le large éventail des œuvres traduites pendant les Lumières espagnoles sera annoncé dans la presse de l'époque. Les principaux journaux (El Mercurio histórico, la Gaceta de Madrid, la Pensadora Gaditana, la Pensadora Salmantina, el Diario de Madrid, el Correo de los ciegos, la Gaceta de Zaragoza, El hablador juicioso...) se firent l'écho de ces nouvelles productions littéraires. Pour plus d'informations, se reporter au travail de Carmen Labrador y Juan Carlos de Pablos Ramírez (1989), La educación en los papeles periódicos de la ilustración española, Madrid: Ministerio de Educación y Ciencia.

17 «CEuvres de la marquise de Lambert pour la bonne éducation morale et politique : traduites à nouveau de la langue française par María Cayetana de la Cerda y Vera, comtesse de Lalaing. On les trouvera à l'imprimerie et librairie de Manuel Martín, calle de la Cruz. » (N.T.)
} 
version espagnole. Après avoir identifié certaines ressemblances que nous exposerons cidessous, nous pouvons imaginer que Cayetana de la Cerda y Vera s'inspira précisément de cette version de 1749 pour réaliser son travail de traduction.

La version originale était composée des différents avertissements provenant des librairies ayant en charge les éditions précédentes, celle de 1747 et celle de 1748, auxquelles s'ajoute un long Abrégé sur la vie de Madame de Lambert. Or toutes ces pages ne furent pas traduites dans la version espagnole. En lieu et place de ces passages, la marquise de Lalaing décida de rédiger un bref prologue et une adresse à Doña Luisa de Borbón, princesse des Asturies et épouse du monarque Charles IV, où elle justifiait son travail de traduction et l'utilité de celui-ci pour la morale et le progrès de la nation espagnole : « La benignidad de V.A., su talento, e instrucción me animan a ponerlo a sus pies, esperando de su notoria bondad lo admita. Yo quisiera poder tributar a V.A. un obsequio digno de su persona; pero a esto no alcanza mi corto talento. $»^{18}$ (LAMBERT, 1781, prologue de la traductrice).

Comme la majorité des femmes-auteures avaient l'habitude de le faire, María Cayetana de la Cerda y Vera rédigea aussi dans son prologue ce captatio benevolentiae, si caractéristique des productions féminines des Lumières, où elle justifiait son travail de traduction en exposant que : « Mucho tiempo hace que deseaba emplear mis ratos desocupados en alguna cosa útil y provechosa, que poder presentar al público $»^{19}$ (LAMBERT, 1781, s/p.) tout en implorant la bienveillance de ces lecteurs éventuels : «No solicito que se celebre mi trabajo; pues no lo escribo buscando alabanzas; me contentaré con que no merezca crítica, y pueda ser útil para el público $»^{20}$ (LAMBERT, 1781, prologue de la traductrice).

María Cayetana de la Cerda y Vera poursuit son prologue en expliquant comment, après plusieurs recherches, elle finit par découvrir une œuvre française, « idioma en que tanto bueno se ha escrito $»^{21}$ (LAMBERT, 1781, prologue de la traductrice) convenant à ses capacités intellectuelles. La traductrice elle-même affirme que lors de ses recherches, elle trouva

\footnotetext{
18 « La bienveillance de S.A., son talent et son instruction m'incitent à lui présenter mon travail, en espérant que sa bonté l'accepte. Je voudrais pouvoir offrir à S.A. un présent digne de sa personne ; mais mon faible talent n'y suffit point. » (N.T.)

${ }^{19}$ « Cela faisait longtemps que je désirais employer mes moments d'oisiveté à une chose utile et profitable, que je pourrais présenter au public. » (N.T.)

${ }^{20}$ « Je ne demande pas d'éloges pour mon travail, puisque je ne l'écris pas en quête de louanges ; je me contenterai de ce qu'il ne me vaille pas de critiques, et qu'il puisse être utile à son public. » (N.T.)

${ }^{21}$ «Une langue dans laquelle tant de choses de qualité ont été écrites. » (N.T.)
} 
plusieurs œuvres qu'elle rejeta finalement, « por ser asuntos superiores a mi corto talento, ya por lo difuso de las Obras $»^{22}$ (LAMBERT, 1781, prologue de la traductrice).

Suite à notre étude comparative, il est étonnant de constater que cette femme de lettres espagnole met elle-même en évidence, dans son prologue, les premières différences entre les deux versions.

Por fin llegaron a mis manos las Obras de la Marquesa de Lambert (Señora muy recomendable por todas circunstancias); y habiendo hallado en ellas unos Tratados sumamente morales, e instructivos, me resolví a traducir los que podrían traer más utilidad, separando algunos, que, aunque muy buenos, no eran el objeto que yo me proponía. ${ }^{23}$ (LAMBERT, 1781, prologue de la traductrice).

Selon la traductrice espagnole, seuls « les passages les plus utiles » furent traduits, ce qui donne, une fois de plus, une version hispanique beaucoup plus réduite que l'original. La comparaison des deux tables des matières met bien en évidence cette modification et la réduction considérable qui en découle :

\begin{tabular}{|c|c|}
\hline $\begin{array}{c}\text { Euvres complètes de Madame la Marquise de } \\
\text { Lambert (1748) }\end{array}$ & Obras de la Marquesa de Lambert (1784) \\
\hline Avis d'une Mère à son Fils & Advertencias de una Madre a su Hijo \\
\hline Avis d'une Mère à sa Fille & Advertencia de una Madre a su Hija \\
\hline Traité de l'Amitié & Tratado de la Amistad \\
\hline Traité de la Vieillesse, à Mlle sa fille & Tratado de la Vejez \\
\hline Réflexions sur les Femmes & Reflexiones nuevas sobre las mujeres \\
\hline Réflexions sur le Goût & Reflexiones sobre el gusto \\
\hline Réflexions sur les Richesses & Reflexiones sobre las Riquezas \\
\hline Psyché, en grec Âme. & Psyche en Griego, Alma \\
\hline
\end{tabular}

\footnotetext{
${ }^{22}$ « Parce qu'elles traitaient de sujets auxquels mon talent ne suffirait pas, à cause de la complexité de ces œuvres. $»($ N.T.).

${ }^{23}$ " Finalement, sont arrivées entre mes mains les œuvres de la marquise de Lambert (une dame très recommandable en tout point) ; et puisque j'y ai trouvé quelques traités extrêmement moraux et instructifs, je me suis résolue à traduire ceux qui pourraient être de la plus grande utilité, tout en omettant certains qui, bien que très bons, ne correspondaient pas au but que je m'étais fixée. » (N.T.)
} 


\begin{tabular}{|c|c|}
\hline $\begin{array}{c}\text { Portraits des diverses personnes : De M. De... de } \\
\text { Melle. De..., de M. de S... et de M. de F... }\end{array}$ & Passages supprimés \\
\hline $\begin{array}{c}\text { Dialogue entre Alexandre et Diogène sur l'égalité } \\
\text { des Biens }\end{array}$ & $\begin{array}{c}\text { Diálogo entre Alejandro y Diógenes sobre la } \\
\text { igualdad de los bienes }\end{array}$ \\
\hline $\begin{array}{c}\text { Discours sur le sentiment d'une Dame, qui croyait } \\
\text { que l'Amour convenait aux Femmes, lors même } \\
\text { qu'elles n'étaient plus jeunes }\end{array}$ & $\begin{array}{c}\text { Discurso sobre el dictamen de una Señora, que } \\
\text { creía, que el amor convenía á las mujeres, aun } \\
\text { cuando ya no eran jóvenes }\end{array}$ \\
\hline $\begin{array}{c}\text { Discours sur la Délicatesse d'Esprit et de } \\
\text { Sentiment }\end{array}$ & $\begin{array}{c}\text { Discurso sobre la delicadez del entendimiento } \\
\text { y de los afectos. }\end{array}$ \\
\hline $\begin{array}{c}\text { Discours sur la différence qu'il y a de la } \\
\text { Réputation à la Considération }\end{array}$ & $\begin{array}{c}\text { Discurso sobre la diferencia que hay de la } \\
\text { Reputación a la Consideración. }\end{array}$ \\
\hline La Femme Hermite & Passages supprimés \\
\hline Lettres diverses & Passages supprimés \\
\hline Supplément à Mme la Marquise de Lambert par \\
Madame Vatry
\end{tabular}

Tableau 3. Table des matières Euvres complètes de Madame la Marquise de Lambert

María Cayetana de la Cerda y Vera décida de faire abstraction de tous les portraits, les lettres et les suppléments des diverses personnes faisant partie de la société française, sans doute en raison du peu d'intérêt qu'ils auraient suscité chez les lecteurs espagnols. Certes, certains passages de l'œuvre d'Anne-Thérèse de Lambert, qui traitaient de thèmes traditionnellement français et visaient un public français, étaient dénués de toute utilité pour les lecteurs hispaniques, raison pour laquelle la traduction de ces passages aurait entraîné d'énormes difficultés pour la compréhension de l'œuvre.

Après la comparaison des deux textes, nous pouvons affirmer que, malgré certaines suppressions incontestables qu'elle choisit d'effectuer, Cayetana de la Cerda réalisa, pour les passages retenus, une traduction assez fidèle à l'œuvre originale. Un fait plutôt rare parmi les traducteurs et les traductions publiés à l'époque, car comme le disait très bien le censeur de l'Académie royale espagnole, Antonio Capmany :

Le respect absolu du sens exact du texte original est une règle qui fut ignorée par de trop nombreux traducteurs qui montraient dans leurs versions espagnoles beaucoup d'imprécisions, qui allaient jusqu'à changer complètement la signification de phrases entières ou, pire encore, 


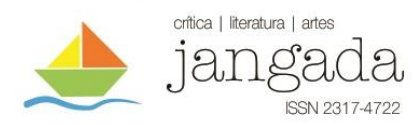

supprimaient même, sans l'indiquer, certains passages. (CAPMANY, 1839, p. $81)$.

La proximité relative des deux versions ne fut pas un obstacle à la compréhension finale du texte. En effet, malgré certaines omissions inévitables, la traductrice fut capable de livrer une version proche de la langue et de la culture espagnoles, en évitant ainsi de faire une traduction littérale trop proche du texte d'origine.

Même si les Euvres complètes de Madame de Lambert obtinrent rapidement « l'avis favorable » inquisitorial, nous avons eu l'impression, au cours de l'analyse de cette traduction, que les intentions à visée éducative de la pédagogue française et celles de la traductrice différaient de celles d'autres pédagogues publiés tout au long du siècle. À la différence d'autres traducteurs des Lumières, qui adaptaient souvent leurs versions aux différents cercles sociaux de l'époque, et malgré l'objectif pédagogique évident de cet ouvrage, Cayetana de la Cerda ne souhaita pas adapter sa traduction aux lecteurs moins instruits. Elle destina plutôt son travail aux femmes et aux hommes les plus cultivés et les plus désireux d'acquérir de nouvelles connaissances (BOLUFER, 2015, p. 26). Cette décision fut, sans doute, l'une des responsables du faible retentissement de son œuvre dans l'ensemble de la société espagnole de l'époque.

\section{MARIE DE RABUTIN-CHANTAL, MARQUISE DE SÉVIGNÉ : LA POPULARITÉ DES RECUEILS ÉPISTOLAIRES.}

Tout au long de ce travail, nous avons pu démontrer que la popularité de la littérature pédagogique était importante dans l'Espagne «ilustrada». Or, toutes les intellectuelles ne connurent pas le même succès ni le même accueil en Espagne, et beaucoup d'entre elles jouirent d'une reconnaissance bien plus tardive. Tel est le cas de Madame de Sévigné, qui connut la célébrité après sa mort, grâce à la publication de sa correspondance avec sa fille. Les lettres de Madame de $S^{* * *}$, qui firent l'objet d'une première édition clandestine en 1725 , comprenaient 28 lettres ou extraits de lettres dans lesquels cette auteure livrait un excellent témoignage de la vie sociale de son époque. En dépit de cela, la réception de son œuvre en Espagne fut beaucoup plus faible et tardive. Alors que nombreuses furent les références journalistiques françaises ${ }^{24}$

\footnotetext{
${ }^{24}$ Le Mercure de France affirmait dans sa publication de mai 1726, que les lettres de l'auteure française ont été « reçues fort agréablement » et qu'on « les lit avec grande avidité ».

Jangada | nr. 12, jul/dez, 2018 | ISSN 2317-4722 


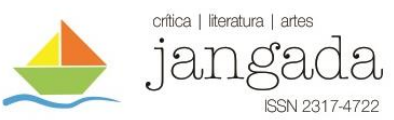

qui louaient le travail épistolaire de l'auteure disparue, nous n'avons retrouvé aucune allusion à la production littéraire de l'écrivaine dans les journaux espagnols des Lumières.

Toutefois, le lecteur espagnol de l'époque eut la possibilité de connaître une œuvre rédigée par le jésuite Juan Andrés pendant son exil italien, dans laquelle celui-ci offrait une première approche du travail de Sévigné. D’après l'homme religieux, Madame de Sévigné possédait un style élégant, naturel et intelligent : il la considérait comme un excellent modèle pour les différents auteurs épistolaires des Lumières espagnoles. Dans son œuvre Origen, progresos y estado actual de toda la literatura (1789), il écrivait : «Pero la soberana maestra y la verdadera reina del estilo epistolar, superior en su género, no solo a las más celebradas mujeres antiguas y modernas, sino también a los más elocuentes franceses, debe llamarse sin contradicción alguna la marquesa de Sévigné. ${ }^{25}$ (ANDRÉS, 1784, pp. 354-355).

Néanmoins, même si les traductions espagnoles de Madame de Sévigné furent postérieures au siècle des Lumières, nous savons que comme dans beaucoup d'autres cas, la production littéraire de la célèbre auteure n'était pas inconnue des intellectuels hispaniques de l'époque. De fait, le livre arriva sous le manteau et, naturellement, dans sa version originale : sa lecture fut une réalité parmi les divers cercles culturels hispaniques.

Le jésuite Juan Andrés affirmait que l'empreinte espagnole de la femme de lettres française fut importante. Il soutenait que, malgré sa faible influence sociale, cette œuvre était très estimée par Charles IV, par exemple, qui la jugeait très appropriée pour l'instruction de son dauphin. Tout comme le roi espagnol, Melchor Gaspar de Jovellanos lut le célèbre recueil entre 1806 et 1807. Grâce au journal intime de ce savant, nous savons qu'il consacrait ses matinées à lire : «Catorce gacetas francesas, escribiendo largo al editor, y notando al Candasín, a quien por fin se envió corregido el soneto, para que le rompiese, pues no salió tan a gusto que fuese a su destino. Se leyó en Cicerón, Policiano y la Sévigné. $»^{26}$ (ESTER, 2002, p. 121).

D'ailleurs, les différentes pages du journal intime de l'intellectuel espagnol nous dévoilent aussi les soupçons inquisitoriaux envers la célèbre œuvre épistolaire de Madame de Sévigné. Après la décision de Jovellanos (grand connaisseur de la langue et de la culture

\footnotetext{
25 « Mais le maître incontestable et la vraie reine du style épistolaire, supérieure dans son genre, non seulement au regard des écrivaines renommées des temps anciens et modernes, mais également à celui de ses contemporains les plus éloquents, doit, sans nul doute, être la marquise de Sévigné. » (N.T.)

26 «Il occupe ses matinées à lire quatorze gazettes françaises, à écrire longuement à l'éditeur et à faire des remarques à Candasín, à qui le sonnet corrigé fut enfin envoyé, mais qui le rejeta, car il n'était pas à son goût. Il a été lu chez Cicéron, Policiano et la Sévigné. » (N.T.)
} 
françaises) de commander ${ }^{27}$ une édition des lettres de l'auteure française, les censeurs se méfièrent de l'intérêt soudain de l'intellectuel pour ce recueil de lettres. Après une analyse exhaustive, ils décidèrent d'interdire la circulation de l'œuvre en laissant entrevoir l'existence d'une certaine relation entre la production de l'auteure française et le jansénisme (LA PARRA LÓPEZ, 1998, p. 121).

Au début du nouveau siècle, l'intérêt suscité par cette écrivaine s'afficha de plus en plus. Même si les lecteurs intéressés ne pouvaient pas encore lire de traduction espagnole de son œuvre, les références qui soulignaient la célébrité des écrits de Sévigné se multiplièrent. En 1803 par exemple, l'écrivain Antonio Marqués y Espejo publia une vaste étude sur la Retórica epistolar, o arte nuevo de escribir todo género de cartas misivas y familiares; con ejemplos de los autores más célebres, extranjeros y nacionales. Cette rhétorique désignait la femme de lettres comme la promotrice d'un nouvel esprit épistolaire, et son auteur décrivait les écrits de la Française en ces termes :

No son las cartas disertaciones, ni poemas; por consiguiente, se discurre en ellas sin argüir, y deben escribirse sin trabajo por llenarlas de buenas frases. Júntese en ellas, con natural arte, el talento y la razón; las máximas, y las chanzas; la sátira aguda y la discreta lisonja. Se habla en ellas de todo; pero no se profundizan las cuestiones para no cansar: solo se proponen como de paso, y se tratan con rapidez. ${ }^{28}$ (MARQUÉS Y ESPEJO, 1803, pp. 33-34).

La première traduction officielle de Madame de Sévigné vers l'espagnol que nous avons pu trouver date de 1818. C'est ici un homme, un religieux, José Marchena, qui signe cette première version en langue castillane. Il faut préciser que la traduction ne fut pas imprimée dans une imprimerie hispanique, mais dans la ville française de Nîmes. La proximité avec la péninsule Ibérique et le nouveau contexte politique ${ }^{29}$ de l'époque rendirent beaucoup plus aisée la réception espagnole de cette première version.

\footnotetext{
${ }^{27}$ Pour plus d'informations, voir la Correspondance de G. M. de Jovellanos du 3 avril 1801 jusqu'en septembre 1808 : Lettre au censeur Juan Pascual de Churruca.

${ }^{28}$ « Il ne s'agit ni de lettres, ni de dissertations, ni de poèmes ; par conséquent, la plume y file, sans argumenter, et les phrases sont écrites sans doute avec la plus grande aisance, au vu des bons mots qui s'y trouvent. Y sont réunis, avec un don naturel, le talent et la raison ; les maximes et les plaisanteries ; la satire mordante et la flatterie discrète. Elles parlent de tout ; mais les questions ne sont pas approfondies afin de ne pas lasser les lecteurs : elles sont seulement évoquées sans qu'on s'y appesantisse, et traitées avec rapidité. » (N.T.)

${ }^{29}$ L'instauration, en 1812, de la première Constitution espagnole met un point final au règne de la terreur, mené par le Saint-Office. L'arrivée d'un nouvel air plus démocratique donna lieu à de nouvelles publications et à une ouverture timide des lettres hispaniques aux cercles culturels étrangers.
} 
Même si les lecteurs espagnols connurent tardivement la production littéraire de la marquise de Sévigné, il faut signaler le nombre important de versions et d'éditions qui suivirent cette première traduction. Les différentes rééditions de Cartas escogidas de Madame de Sévigné acompañadas de notas explicativas sobre los hechos y las personas de su tiempo, precedidas de observaciones literarias por Mr. de Sainte-Beuve sont encore d'actualité aujourd'hui, puisque la dernière édition de la marquise de Sévigné date de 2006.

\section{CONCLUSION}

En définitive, la réception espagnole des œuvres de Louise d'Épinay, Anne-Thérèse de Lambert ou Marie de Rabutin-Chantal, dite Madame de Sévigné, constitue un chapitre important de la fortune littéraire que ces auteures connurent en dehors des frontières françaises et plus concrètement dans l'Espagne des Lumières. Tout au long de ce travail, nous avons pu démontrer comment l'obsession pédagogique des intellectuels des Lumières espagnoles, l'intérêt des femmes pour la lecture et le développement du monde éditorial, ont produit pendant tout le XVIII ${ }^{\mathrm{e}}$ siècle une véritable avalanche de textes destinés à la formation et à l'instruction féminine - et ce notamment vers le milieu du siècle, lorsqu'un fort intérêt pour les traductions d'œuvres étrangères se fait sentir. En harmonie avec cet intérêt pédagogique du siècle, l'éducation devient donc l'un des sujets phare de la production éditoriale de l'époque. La visée pédagogique des productions littéraires de ces trois pédagogues françaises séduit un grand nombre de lecteurs, notamment des lectrices, au cours des Lumières espagnoles, car la sensibilité de ces créatrices en matière de morale et de religion s'adaptait à la perfection aux créations littéraires de l'Espagne des Lumières. Il s'agit ainsi d'une littérature respectueuse des valeurs spirituelles traditionnelles, et en même temps ouverte au nouveau concept de « vertu sensible ».

\section{RÉFÉRENCES BIBLIOGRAPHIQUES}

AGUILAR PIÑAL, F. Índice de las poesías publicadas en los periódicos españoles del siglo XVIII. Madrid: C.S.I.C., 1981.

AMAR Y BORBÓN, J. Discurso sobre la educación física y moral de las mujeres. Madrid: Benito Cano, 1790.

ANDRÉS, J. Origen, progresos y estado actual de toda la literatura. Madrid: Aduana Vieja, 1784.

Jangada | nr. 12, jul/dez, 2018 | ISSN 2317-4722 


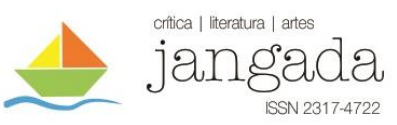

BOLUFER PERUGA, M. Espectadores y lectoras: representaciones e influencia del público femenino en la prensa del siglo XVIII. In: Separata de Cuadernos de Estudio del siglo XVIII. Oviedo, $n^{\circ}$ 5, pp. 23-57, 1995.

Pedagogía y moral en el siglo de las Luces: las escritoras francesas y su recepción

en España. In: Revista de historia moderna. Alicante, $\mathrm{n}^{\circ}$ 20, pp. 251-292, 2002.

Una ética de la excelencia: Cayetana de la Cerda y la circulación de Madame de Lambert en España. In: Cuadernos de historia moderna. Madrid, n 40, pp. 241-264, 2015.

CAPEL MARTÍNEZ, R.M ${ }^{a}$. Prensa y Escritura Femenina en la España Ilustrada. In: $E l$ $\begin{array}{lllll}\text { argonauta español. } & \text { Aix-en-Provence, } & \mathrm{n}^{\circ} & 7, & 2010 .\end{array}$ https://journals.openedition.org/argonauta/431 (Accès à : 12, janvier, 2019).

CAPMANY, A. de. Arte de traducir el idioma francés al castellano. Madrid: Antonio de Sancha, 1776.

ÉPINAY, L. de. Las conversaciones de Emilia. Madrid: Benito Cano, 1797. . Les Conversations d'Émilie. Paris : Humblot, 1781.

ESTER, J. Recepción de autores franceses de la época clásica en los siglos XVIII y XIX en España y en el extranjero. Madrid: UNED, 2002.

GARCÍA HURTADO, M.R. «La traducción en España, 1750-1808: cuantificación y lenguas en contacto ». In: LAFARGA, F. La Traducción en España (1750-1830): lengua, literatura, cultura. Lleida: Edicions de la Universitat de Lleida, 1999.

GENLIS, F. de. Veladas de la Quinta o Historias sumamente útiles para las madres de familia. Madrid : Manuel González, 1788.

JOVELLANOS, G.M. Correspondance de G. M. de Jovellanos du 3 avril 1801 à septembre 1808. Lettre au censeur Juan Pascual de Churruca. Oviedo: Institut Feijoo, 1988.

LA PARRA LÓPEZ, E. Ilustrados e Inquisición ante la Iglesia constitucional francesa. In: Revista de Historia das Ideas. Coímbra, nº 10, pp. 359-374, 1998.

LABRADOR, C., RAMÍREZ, J.C. La educación en los papeles periódicos de la ilustración española. Madrid: Ministerio de Educación y Ciencia, 1989.

LAMBERT, A.T. de. Obras de la marquesa de Lambert. Madrid: Manuel Marín, 1781.

Euvres complètes de Madame la Marquise de Lambert, Réflexions nouvelles sur les femmes, Par une Dame de la Cour. Paris : François Breton, 1727.

MARQUÉS Y ESPEJO, A. Retórica epistolar, o Arte nuevo de escribir todo género de cartas misivas y familiares, con ejemplos de los autores más célebres, extranjeros y nacionales. Madrid : Cruzado, 1803.

MÉRIMÉE, P. L'influence française en Espagne au XVIII ${ }^{e}$ siècle. Paris : Les Belles Lettres, 1936.

MONTERO PIZARRO, R. La pedagogía del terror católico: la Santa Inquisición murciana y el adventismo alicantino. Alicante: Club Universitario, 2005.

MURIEL, A. Historia del reinado de Carlos IV. Madrid: Academia de la Historia, 1894.

PALACIOS, E. La mujer y las letras en el siglo XVIII. Madrid: Laberinto, 2002.

VARGAS PONCE, J. Declaración contra los abusos introducidos en el castellano. Madrid: Ibarra Viuda, 1793. 
VÁZQUEZ, L. Censure de la littérature française dans l'Espagne éclairée. In : Littérales «La Bibliothèque est en feu ». Université de Paris X-Nanterre, nº 8, pp. 29-43, 1991.

VÁZQUEZ-AYORA, G. Introducción a la traductología. Georgetown: University School of Languages and Linguistics, 1977.

Presse périodique :

Gaceta de Madrid. Madrid: Real, 1791.

Gaceta de Madrid. Madrid: Real, 1797.

La Miscelánea instructiva curiosa y agradable o Anales de literatura, ciencias y Artes. Madrid: Antonio Cruzado, 1797.

Memorial literario, instructivo y curioso de la corte de Madrid. Madrid: Real, 1781.

Mercure de France, journal littéraire et politique. Paris : Cailleau, 1733.

Mercure de France, journal littéraire et politique. Paris : Cailleau, 1726.

Mercurio histórico y político de España. Madrid: Real, 1789 